Research article

\title{
Mosquito immune responses and compatibility between Plasmodium parasites and anopheline mosquitoes
} Giovanna Jaramillo-Gutierrez ${ }^{1}$, Janneth Rodrigues ${ }^{1}$, Georges Ndikuyeze ${ }^{1}$, Michael Povelones ${ }^{2}$, Alvaro Molina-Cruz ${ }^{1}$ and Carolina Barillas-Mury*1

Address: ${ }^{1}$ Laboratory of Malaria and Vector Research, National Institute of Allergy and Infectious Diseases, National Institutes of Health, Rockville, MD 29892, USA and 2Immunology and Infection, Division of Cell and Molecular Biology, Faculty of Natural Sciences, Imperial College London, London, UK

Email: Giovanna Jaramillo-Gutierrez - gutierrgi@niaid.nih.gov; Janneth Rodrigues - rodriguesj@niaid.nih.gov; Georges Ndikuyeze - gndiku1@towson.edu; Michael Povelones - m.povelones@imperial.ac.uk; Alvaro Molina-Cruz - amolinacruz@niaid.nih.gov; Carolina Barillas-Mury* - cbarillas@niaid.nih.gov

* Corresponding author

Published: 30 July 2009

BMC Microbiology 2009, 9:154 doi:10.1 |86/147|-2/80-9-154
Received: 18 December 2008

Accepted: 30 July 2009

This article is available from: http://www.biomedcentral.com//47/-2/80/9//54

(c) 2009 Jaramillo-Gutierrez et al; licensee BioMed Central Ltd.

This is an Open Access article distributed under the terms of the Creative Commons Attribution License (http://creativecommons.org/licenses/by/2.0), which permits unrestricted use, distribution, and reproduction in any medium, provided the original work is properly cited.

\begin{abstract}
Background: Functional screens based on dsRNA-mediated gene silencing identified several Anopheles gambiae genes that limit Plasmodium berghei infection. However, some of the genes identified in these screens have no effect on the human malaria parasite Plasmodium falciparum; raising the question of whether different mosquito effector genes mediate anti-parasitic responses to different Plasmodium species.

Results: Four new An. gambiae (G3) genes were identified that, when silenced, have a different effect on P. berghei (Anka 2.34) and P. falciparum (3D7) infections. Orthologs of these genes, as well as LRIMI and CTL4, were also silenced in An. stephensi (Nijmegen Sda500) females infected with $P$. yoelii (I7XNL). For five of the six genes tested, silencing had the same effect on infection in the $P$. falciparum-An. gambiae and $P$. yoelii-An. stephensi parasite-vector combinations. Although silencing LRIMI or CTL4 has no effect in An. stephensi females infected with P. yoelii, when An. gambiae is infected with the same parasite, silencing these genes has a dramatic effect. In An. gambiae (G3), TEPI, LRIMI or LRIM2 silencing reverts lysis and melanization of $P$. yoelii, while CTL4 silencing enhances melanization.

Conclusion: There is a broad spectrum of compatibility, the extent to which the mosquito immune system limits infection, between different Plasmodium strains and particular mosquito strains that is mediated by TEPI/LRIMI activation. The interactions between highly compatible animal models of malaria, such as P. yoelii (I7XNL)-An. stephensi (Nijmegen Sda500), is more similar to that of $P$. falciparum (3D7)-An. gambiae (G3).
\end{abstract}

\section{Background}

Mosquitoes transmit many infectious diseases, including malaria, lymphatic filariasis, yellow fever, and dengue. Among these diseases, malaria is by far the most costly in terms of human health. It is endemic to more than 100 countries and causes 550 million cases per year, with the highest mortality in children from sub-Saharan Africa. Malaria transmission to humans requires a competent 
mosquito species, as Plasmodium parasites must undergo a complex developmental cycle and survive the defense responses of their insect host. In Africa, Anopheles gambiae is the major vector of Plasmodium falciparum infection, which causes the most aggressive form of human malaria.

The Plasmodium berghei (murine malaria) model is one of the most widely used experimental systems to study malaria transmission. Gene silencing by systemic injection of double-stranded RNA (dsRNA) has proven to be a very useful tool to carry out functional genomic screens aimed at identifying mosquito genes that mediate antiparasitic responses. In general, Anopheles gambiae is considered to be susceptible to $P$. berghei infection, because a high prevalence of infection can be achieved and parasites are only rarely melanized; however, silencing of either thioester-containing protein 1 (TEP1) [1], leucine-rich repeat immune protein 1 (LRIM1) [2], or LRIM2 (also called APL1, [3]), enhances $P$. berghei infection by 4-5 fold; indicating that, when these effector molecules are present, about $80 \%$ of parasites are eliminated by a lytic mechanism[1]. It is well documented that An. gambiae mosquitoes have a different transcriptional response to infection with $P$. berghei and $P$. falciparum $[4,5]$ and genes such as LRIM1 and C-type lectin 4 (CTL4) [2], which limit or enhance $P$. berghei infection, respectively, do not affect P. falciparum infection in An. gambiae [6]. This raises the possibility that some antiplasmodial genes identified using the $P$. berghei malaria model may not be relevant to human malaria transmission.

More than 400 species of anopheline mosquitoes have been identified, but only 40 of them are considered to be important disease vectors [7]. Different anopheline species and even particular strains of mosquitoes vary widely in their susceptibility to infection with a given Plasmodium parasite species. For example, twelve different strains of Anopheles stephensi have been shown to have very different susceptibility to $P$. falciparum (Welch strain) infection [8]. Furthermore, susceptibility had a strong genetic component, which allowed selection of a An. stephensi strain (Nijmegen Sda500) that is highly susceptible to $P$. falciparum infection [8]. A strain of An. gambiae (L35) was selected to be highly refractory to infection with Plasmodium cynomolgy (primate malaria). The L35 strain melanizes $P$. cynomolgy, as well as several other Plasmodium species such as $P$. berghei (murine malaria), Plasmodium gallinaceum (avian malaria), and other primate malaria parasites such as Plasmodium gonderi, Plasmodium inui, and Plasmodium knowlesi. Interestingly, P. falciparum strains from the New World are also melanized effectively, but not those of African origin, suggesting that there are genetic differences between $P$. falciparum strains that affect their ability to infect An. gambiae [9]. The African strains of $P$. falciparum tested appeared to be better adapted to their natural mosquito vector. However, great differences in the level of resistance to $P$. falciparum infection have been documented in families derived from individual $A n$. gambiae females collected in the field $[3,10]$, and a small region of chromosome $2 \mathrm{~L}$ is a major determinant of genetic resistance to infection [3].

Drosophila melanogaster can support the development of Plasmodium gallinaceum oocysts when cultured ookinetes are injected into the hemocele [11]. This observation opened the possibility of using a genetic approach to screen for Drosophila genes that affect Plasmodium P. gallinaceum infection[12]. Furthermore, silencing of orthologs (or family members) of five of these candidate genes in An. gambiae (G3 strain) demonstrated that four of them also affected $P$. berghei infection in the mosquito [12].

In this study we compare how silencing a set of genes identified in the Drosophila screen affects Plasmodium infection in different vector-parasite combinations. We conclude that there is a broad range of compatibility between different Plasmodium strains and particular mosquito strains that is determined by the interaction between the parasite and the mosquito's immune system. We define compatibility as the extent to which the immune system of the mosquito is actively limiting Plasmodium infection. For example, the P. yoelii-An. stephensi and $P$. falciparum-An. gambiae strains used in this study are highly compatible vector-parasite combinations, as silencing several genes involved in oxidative response or immunity has no significant effect on infection. In contrast, silencing the same genes has a strong effect in less compatible vector-parasite combinations such as $P$. yoeliiAn. gambiae or P. berghei-An. gambiae.

\section{Results and discussion Effect of GSTTI and GSTT2 silencing on P. berghei infection}

The effect of silencing An. gambiae orthologs (or homologs) of genes originally identified in the Drosophila genetic screen on $P$. berghei infectivity is summarized in Table 1[12]. Knockdown of arginine kinase (ArgK) and oxidation resistance gene 1 (OXR1) reduces infection. Tetraspanin and heat-shock cognate 3 (Hsc-3) silencing have the opposite effect, enhancing infection, while reducing the expression of the solute transporter (Sol. Trsp.) gene did not affect infection with $P$. berghei [12]. The effect of silencing two An. gambiae homologs of a glutathione Stransferase of the theta class (GSTT) (CG1702-PA) gene also identified in the Drosophila screen on $P$. berghei infection was evaluated. Injection of GSTT1 (AGAP000761PA) or GSTT2 (AGAP000888-PA) dsRNA reduced mRNA expression by $60 \%$ and $55 \%$, respectively, relative to the control groups injected with dsLacZ. Both GSTT1 and 
GSTT2 knockdown significantly reduce $P$. berghei infection $(\mathrm{P}<0.05$ and $\mathrm{P}<0.03$, respectively) using the Kolmogorov-Smirnov (KS) test (Figure 1 and Table 1).

\section{Direct comparison of the effect of silencing seven An.} gambiae genes on $\mathbf{P}$. berghei and $\mathbf{P}$. falciparum infection The effect of reducing expression of the five genes previously reported [12] as well as GSTT1 and GSTT2 in An. gambiae infected with $P$. falciparum (3D7 strain) was evaluated (Figure 2). Silencing of ArgK and Hsc-3 significantly reduced infection $(\mathrm{P}<0.05$ and $\mathrm{P}<0.001$, respectively, using the KS test) (Figure 2A, B). Sol. Trsp., GSTT1, and OXR1 silencing did not affect $P$. falciparum infection (Figure 2C-E), while tetraspanin and GSTT2 knockdown enhanced infection ( $\mathrm{P}<0.01$ and $\mathrm{P}<0.03$; KS test) (Figure $2 \mathrm{~F}, \mathrm{G})$. A summary of these results is shown in Table 1.

Silencing ArgK, Sol. Trsp., and tetraspanin genes has a similar effect on $P$. berghei and P. falciparum infection. ArgK is a key enzyme in cellular energy homeostasis in arthropods, with a function similar to that of creatine kinase in mammals. This enzyme catalyzes the synthesis of phosphoarginine, which serves as an energy reserve. The highenergy phosphate in phosphoarginine can be transferred to ADP to renew ATP during periods of high energy demand [13]. Apparently, silencing this enzyme results in
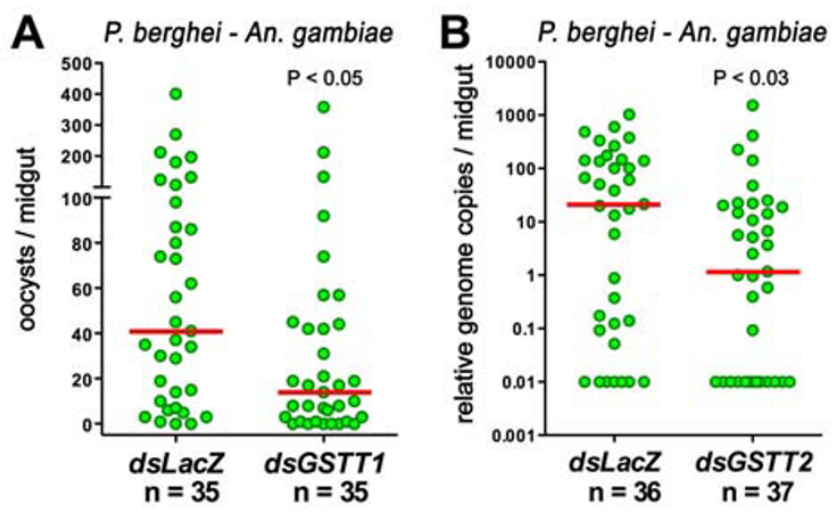

\section{Figure I}

Effect of silencing An. gambiae (G3) GSTTI and GSTT2 on $P$. berghei infection. Panel A, Effect of silencing glutathione-S-transferase theta-I (GSTTI) on Plasmodium infection. GFP-expressing parasites were counted directly 6 days post infection (PI). Panel B, Effect of silencing glutathione-S-transferase theta-2 (GSTT2) on Plasmodium infection. Infection levels were determined based on the relative abundance of $P$. berghei $28 \mathrm{~S}$ and An. gambiae S7 genes in genomic DNA isolated from midguts 6 days PI. The dots represent the infection level on individual midguts, and the median infection level is indicated by the horizontal line. Distributions are shown using a logarithmic scale for GSTT2 and are compared using the Kolmogorov-Smirnov (KS) test; $n=$ number of mosquitoes; $\mathrm{P}$ values lower than 0.05 are considered to be significantly different. a physiologic state in the mosquito that does not foster the development of either $P$. berghei or $P$. falciparum. Silencing of the solute transporter has no effect, while knockdown of tetraspanin enhances infection with both parasites. Tetraspanins are proteins with four transmembrane (TM) domains that are associated extensively with one another and with other membrane proteins to form specific microdomains distinct from lipid rafts. They are expressed on the surface of numerous cell types and are involved in diverse processes from cell adhesion to signal transduction and some of them inhibit the function of other members of the same family of proteins [14]. CD81 is a tetraspanin that has been shown to be required for hepatocyte invasion by $P$. falciparum and $P$. yoelii sporozoites [15]. Silencing of the An. gambiae tetraspanin gene may enhance parasite invasion and/or prevent the activation of an immune cascade that limits infection with $P$. berghei and $P$. falciparum.

OXR1, GSTT1, GSTT2 and Hsc-3 silencing has a different effect on $P$. berghei and $P$. falciparum infection. In yeast and mammals, OXR1 is induced by heat and oxidative stress and prevents oxidative damage by an unknown mechanism [16]. In An. gambiae, OXR1 silencing decreases resistance to oxidative challenge and prevents the induction of genes involved in ROS detoxification, such as catalase, following a blood meal (G. JaramilloGutierrez and C. Barillas-Mury, unpublished). We have previously shown that higher ROS levels in An. gambiae reduce $P$. berghei infection [17]. Thus, it is likely that the decrease in $P$. berghei infectivity following OXR1 silencing is due to an increase in ROS. The unexpected observation that OXR1 silencing does not affect $P$. falciparum infection suggests that either this parasite species is less susceptible to oxidative stress or that the ingestion of human blood results in less accumulation of ROS in the mosquito.

GSTs play an important role as antioxidants and are involved in the detoxification of xenobiotics. GSTs of the epsilon and delta class have been extensively studied for their role in insecticide resistance in mosquitoes [18]. The GST-Theta1 (GSTT1) null genotype in human males is highly associated to increased risk of basal cell carcinoma of the skin [19]. Furthermore, in diabetics, the deletion of one copy of the GSTT1 gene is associated with elevated markers of inflammation and lipid peroxidation [20]. Therefore, silencing of GSTT1 and GSTT2 could result in increased lipid peroxidation, which is expected to be deleterious to $P$. berghei; however, it is not clear why reducing GSTT2 expression enhances $P$. falciparum infection.

\section{Susceptibility of An. stephensi (Nijmegen Sda500 strain) and An. gambiae (G3) to P. yoelii infection}

The observed differences in the effect of silencing specific An. gambiae (G3 strain) genes on P. berghei and P. falci- 
Table I: Effect of silencing seven An. gambiae genes or their orthologs in An. stephensi on the intensity of $P$. berghei, $P$. falciparum or $P$. yoelii infection.

\begin{tabular}{|c|c|c|c|c|}
\hline An. gambiae Gene ID & Gene & An. gambiae $P$. berghei $\left(21^{\circ} \mathrm{C}\right)$ & An. gambiae $P$. falciparum $\left(26^{\circ} \mathrm{C}\right)$ & An. stephensi P. yoelii $\left(24^{\circ} \mathrm{C}\right)$ \\
\hline AGAP005627 & $\operatorname{ArgK}$ & Decreasel & Decrease & \\
\hline AGAPOI 0892 & Sol. trsp. & No effect ${ }^{1}$ & No effect & \\
\hline AGAP005233 & Tetrasp. & Increase & Increase & \\
\hline AGAP00I75I & $O X R I$ & Decreasel & No effect & No effect \\
\hline AGAP004192 & Hsc-3 & Increase & Decrease & Increase \\
\hline AGAP00076 I & GSTTI & Decrease & No effect & No effect \\
\hline AGAP000888 & GSTT2 & Decrease & Increase & Increase \\
\hline AGAP006348 & LRIMI & Increase $^{2}$ & No effect. ${ }^{3}$ & No effect \\
\hline AGAP005335 & CTL4 & Decrease $^{2}$ & No effect. ${ }^{3}$ & No effect \\
\hline
\end{tabular}

'Brandt et al., 2008

2Osta et al., 2004

${ }^{3}$ Cohuet et al., 2006

parum infection may reflect the degree of compatibility between these two parasite species and the mosquito strain used. Alternatively, mosquitoes may trigger different sets of effector genes in response to different Plasmodium species. To explore these possibilities, we evaluated the responses of two mosquito species that differ in their susceptibility to the same Plasmodium parasite.

The susceptibility of An. stephensi (Nijmegen Sda500), a strain highly susceptible to $P$. falciparum infection [8], and An. gambiae (G3) females to $P$. yoelii infection was compared by feeding them on the same infected mouse. An stephensi is highly susceptible to $P$. yoelii infection, as no melanized parasites are observed and the median number of live oocysts is 51-fold higher than in An. gambiae (Figure $3 \mathrm{~A}, \mathrm{C}$ and Table 2). In contrast, An. gambiae (G3) is partially refractory and has two distinct phenotypes (Figure $3 \mathrm{~B}$ ). In approximately half of the mosquitoes, all parasites are melanized, while in the other half, parasite lysis appears to be the main defense response, as no melanizations are observed (Figure 3C, D). Interestingly, the prevalence of mixed phenotypes-that is, mosquitoes in which both live and melanized parasites are observed-is low (10\%; Table 2). These results are in agreement with a previous report in which susceptibility of An. gambiae (G3) and An. stephensi (Pakistan) to P. yoelii infection was compared [21].

\section{Effect of silencing An. stephensi orthologs on P. yoelii infection}

Six genes whose phenotypes differ when An. gambiae is infected with $P$. berghei or $P$. falciparum were examined. An. stephensi orthologs of OXR1, Hsc-3, GSTT1, and GSTT2, as well as two other genes previously reported in the literature (LRIM1 and CTL4), were silenced, and the effect on $P$. yoelii infection was evaluated. Five of the six genes tested had similar effects in the An. gambiae-P. falciparum and the An. stephensi-P. yoelii systems (Table 1).
Silencing OXR1, LRIM1, CTL4, or GSTT1 had no effect, while GSTT2 and Hsc-3 silencing enhanced $P$. yoelii infection in An. stephensi (Figure 4 and Table 1). Hsc-3 was the only gene that gave a different phenotype between $A n$. gambiae-P. falciparum and An. stephensi-P. yoelii. Conversely, this was also the only gene that had a similar phenotype in An. gambiae infected with P. berghei and in $P$. yoelii-infected An. stephensi. The expression of heat shock proteins is temperature dependent; thus the differences in the effect of Hsc-3 silencing in mosquitoes infected with different Plasmodium species could be due to physiologic differences resulting from the temperature at which infected mosquitoes are kept. For example, Hsc-3 silencing decreases P. falciparum infection $\left(26^{\circ} \mathrm{C}\right)$ in $\mathrm{An}$. gambiae but results in a significant but mild increase in $P$. yoelii infection $\left(24^{\circ} \mathrm{C}\right)$ in $A n$. stephensi and a strong enhancement of $P$. berghei infection $\left(21^{\circ} \mathrm{C}\right)$ in An. gambiae. Interestingly, a decrease in parasite number is also observed in the Drosophila line in which a P-element has been inserted close to the $H s c-3$ gene. In the fly system, in vitro cultured $P$. gallinaceum ookinetes are injected into the hemocele and the infected flies kept at $27^{\circ} \mathrm{C} \mathrm{[12].} \mathrm{It} \mathrm{appears} \mathrm{that}$ silencing $\mathrm{Hsc}$-3 decreases Plasmodium infection when the infected insects are kept at a higher temperature but has the opposite effect, enhancing infection, when infected insects are kept at a lower temperature.

\section{Refractoriness of An. gambiae (G3) to P. yoelii infection is due to activation of the mosquito immune system} The fact that LRIM1 and CTL4 silencing in An. stephensi (Nijmegen Sda500 strain) had no effect on $P$. yoelii infection could reflect a lack of activation of the immune system in this highly susceptible mosquito strain. Alternatively, it is also possible that LRIM1 and CTL4 do not participate in mosquito antiparasitic responses to $P$. yoelii. To explore these two possibilities, the effect of CTL4 and LRIM1 silencing in An. gambiae (G3) females, which are partially refractory to $P$. yoelii infection, was investi- 

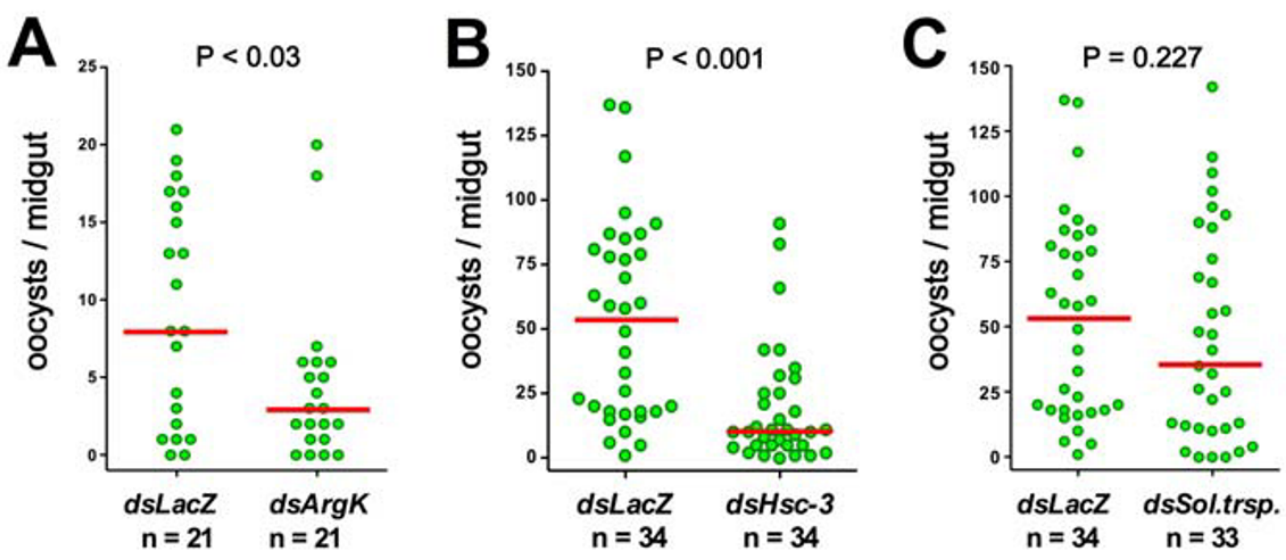

\section{P. falciparum An. gambiae}
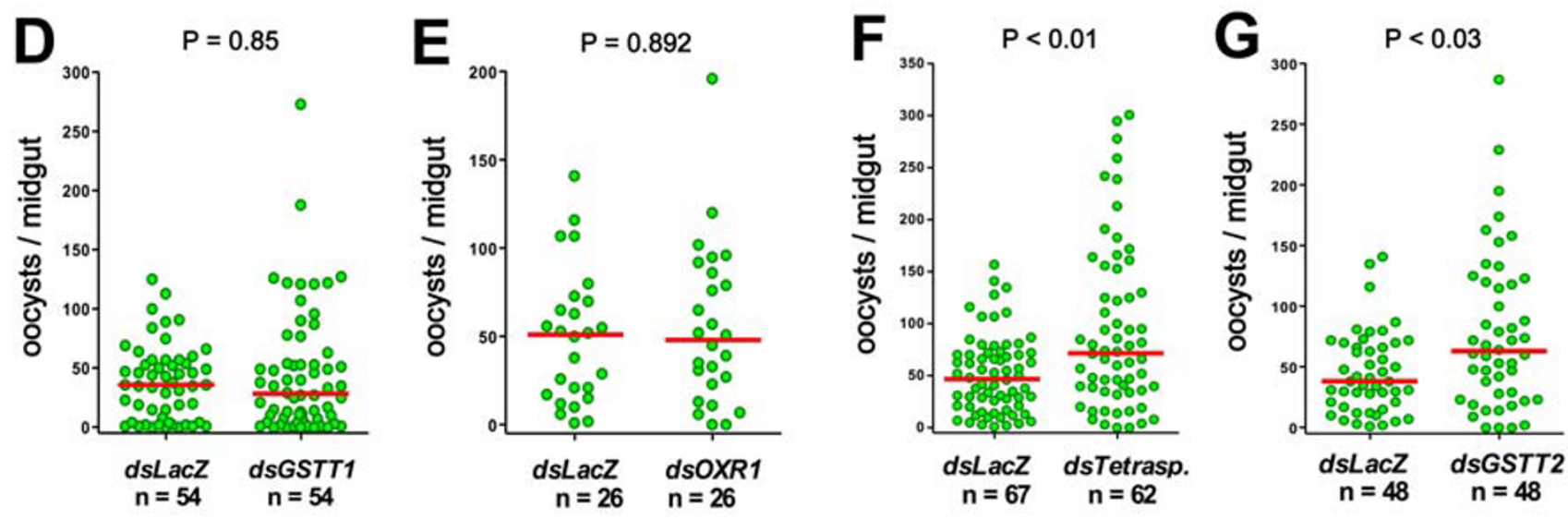

\section{Figure 2}

Effect of silencing several An. gambiae (G3) genes on parasite $P$. falciparum infection. Effect of silencing arginine kinase (ArgK) (Panel A), heat shock cognate 3 (Hsc-3) (Panel B), solute transporter (Sol. Trsp.) (Panel C), glutathione-S-transferase theta-I (GSTTI) (Panel D), oxidation resistance gene I (OXRI) (Panel E) tetraspanin (Tetrasp.) (Panel F), and glutathioneS-transferase theta-2 (GSTT2) (Panel G) on $P$. falciparum infection. The number of $P$. falciparum oocysts present was determined by directly counting mercurochrome-stained parasites 7-8 days post infection. The dots represent the number of parasites present on individual midguts, and the median number of oocysts is indicated by the horizontal line. Distributions are compared using the Kolmogorov-Smirnov test; $n=$ number of mosquitoes; $P$ values lower than 0.05 are considered to be significantly different.

gated. CTL4 silencing increases the number of melanized parasites from $62 \%$ to $95 \%$ (Figure 3A). Conversely, LRIM1 silencing completely reverts $P$. yoelii melanization and increases the median number of live oocysts by 4.6 fold (Figure 5B). To further investigate the participation of the An. gambiae immune system on the partial refractoriness of this species to $P$. yoelii infection, the effect of silencing TEP1 and LRIM2 was also evaluated. TEP1 and LRIM2 had a similar effect as LRIM1, enhancing infection by 32 and 20.5 fold, respectively (Figure 5C, D).

\section{Conclusion}

The effect of silencing multiple mosquito genes in the highly compatible $P$. yoelii (17XNL)-An. stephensi (Nijmegen Sda500)system was very similar to that observed when P. falciparum (3D7) was used to infect An. gambiae (G3), its natural vector; suggesting that $P$. yoeliiAn. stephensi is a representative animal model to study $P$. falciparum interactions with compatible vectors. Furthermore, $P$. yoelii-infected females can be kept at $24^{\circ} \mathrm{C}$, a temperature that is more physiological for mosquitoes and closer to that used for $P$. falciparum infections $\left(26^{\circ} \mathrm{C}\right)$.

Using less compatible parasite-mosquito combinations, such as the P. berghei-An. gambiae or P. yoelii-An. gambiae strains described in this study, may be particularly useful to identify and characterize immune pathways in the mosquito that could potentially limit human malaria transmission. Once a potential pathway is defined, it is possible to investigate if certain parasite strains avoid acti- 
P. yoelii - An. stephensi
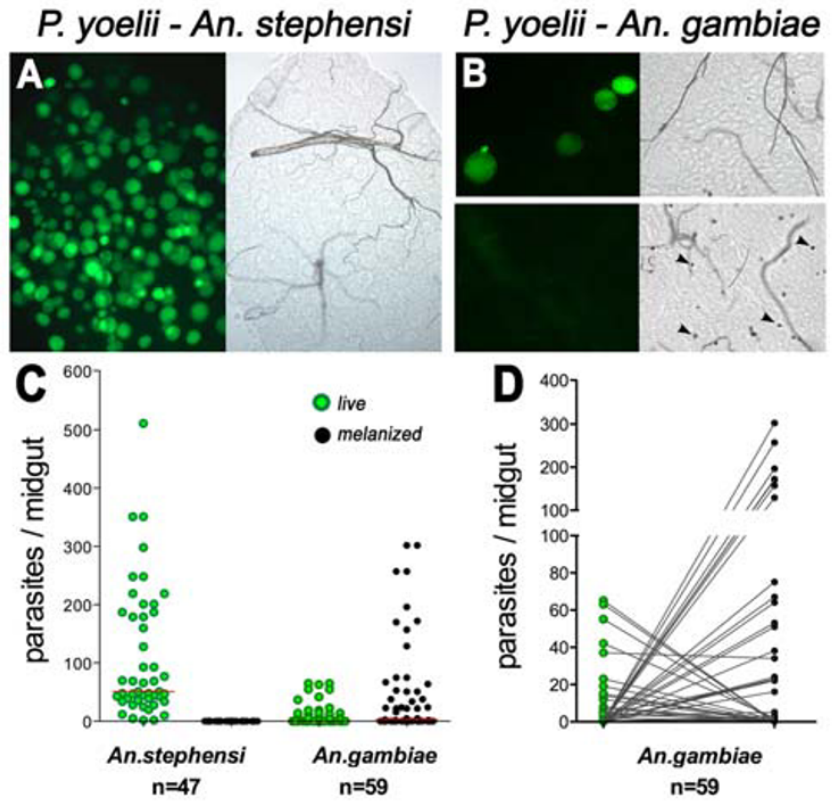

Figure 3

Susceptibility of An. stephensi (Nijmegen Sda500) and An. gambiae (G3) to $P$. yoelii infection. An. stephensi and An. gambiae mosquitoes were fed on the same $P$. yoeliiinfected mouse. The images illustrate the level of infection and parasite melanization observed 6 days post infection (PI) in An. stephensi (Panel A) or An. gambiae (Panel B) females infected with $P$. yoelii. Live parasites are detected with green fluorescence (left panels), and those melanized are in DIC images (right panels). Panel C, Number of live (green dots) or melanized (black dots) parasites present on individual midguts 6 days PI. The median number of oocysts is indicated by the horizontal line. Distributions are compared using the Kolmogorov-Smirnov test; $\mathrm{n}=$ number of mosquitoes; $\mathrm{P}$ values lower than 0.05 are consider to be significantly different. Panel D, The number of live (green dots) and melanized (black dots) $P$. yoelii parasites on individual An. gambiae midguts is shown connected by a line. In most mosquitoes, either all parasites are alive or all are melanized. There are very few midguts in which both live and melanized parasites are observed. vating them, or if the effector genes are inefficient. It may also be possible to use alternative strategies (such as chemicals or fungal infections) to activate these potential antiplasmodial responses and test their effectiveness in limiting malaria transmission in natural vector-parasite combinations.

There is a broad spectrum of compatibility between different strains of Plasmodium and particular mosquito strains; for example, An. gambiae (G3) is highly compatible with P. falciparum (3D7) parasites, but has low compatibility with $P$. yoelii $17 \mathrm{XNL}$. A given strain of Plasmodium can also be more compatible with certain mosquitoes. For example, P. yoelii 17XNL is much more compatible with An. stephensi (Nijmegen Sda500 strain) than with An. gambiae (G3). TEP1 silencing in An. gambiae (Keele strain) mosquitoes enhances infection with $P$. falciparum (NK54 strain), doubling the median number of oocysts [22]. Silencing TEP1 in An. gambiae has a more dramatic effect (4-5 fold increase) on $P$. berghei infection [1]. Furthermore, silencing TEP1 in An. gambiae (G3 strain) does not enhance infection with $P$. falciparum (NF54 strain), indicating that there are differences in compatibility between particular strains of An. gambiae and P. falciparum (M. Povelones and A. Molina-Cruz, unpublished).

Over activation of the Rel2 pathway by silencing Caspar, a critical suppressor of this cascade, drastically reduces $P$. falciparum (NK54 strain) infection in An. gambiae (Keele strain), An. albimanus (Santa Tecla strain) and An. stephensi mosquitoes [22]. Double silencing experiments in $A n$. gambiae (Keele strain) females, in which Caspar and TEP1 (or other effectors of the Rel2 pathway) were co-silenced, rescues the effect of Caspar, indicating that TEP1 is an important effector of this response. The fact that strong activation of the Rel2 pathway can very effectively prevent infection in several mosquito species that are natural vectors of P. falciparum [22], begs the question of why this immune response is not effective preventing disease transmission under natural field conditions.

Table 2: An. gambiae (G3) and An. stephensi (Nijmegen Sda500) infections with P. yoelii.

\begin{tabular}{|c|c|c|c|c|c|}
\hline Mosquito species & $\begin{array}{l}\text { Prevalence of } \\
\text { infection }\end{array}$ & $\begin{array}{c}\text { Median live oocyst } \\
\text { number }\end{array}$ & Oocyst range & $\begin{array}{l}\% \text { of midguts with } \\
\text { melanized parasites }\end{array}$ & $\begin{array}{c}\% \text { of midguts with live } \\
\text { and melanized } \\
\text { parasites }\end{array}$ \\
\hline $\begin{array}{c}\text { An. gambiae } \\
\mathrm{n}=59\end{array}$ & $52 \%$ & 1 & $0-65$ & $59 \%$ & $10 \%$ \\
\hline $\begin{array}{c}\text { An. stephensi } \\
\mathrm{n}=47\end{array}$ & $100 \%$ & 51 & $2-302$ & $0 \%$ & $0 \%$ \\
\hline
\end{tabular}




\section{P. yoelii - An. stephensi}
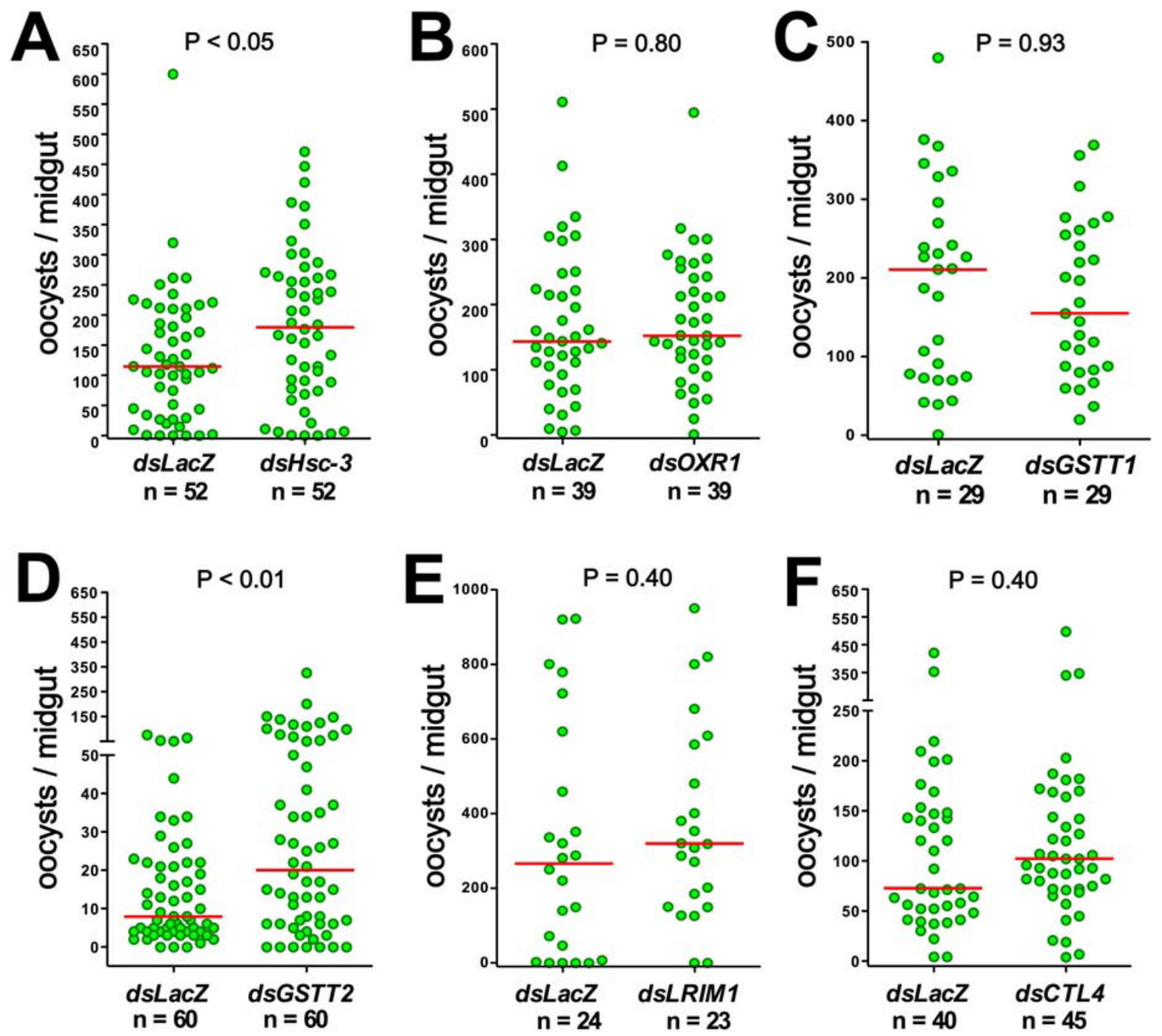

Figure 4

Effect of silencing several An. stephensi (Nijmegen Sda500) genes on $P$. yoelii infection. Effect of silencing heat shock cognate 3 (Hsc-3) (Panel A), oxidation resistance gene (OXRI) (Panel B), glutathione-S-transferase theta-I (GSTTI) (Panel C), glutathione-S-transferase theta-2 (GSTT2) (Panel D), leucine rich-repeat immune protein I (LRIMI) (Panel E), and C-type lectin 4 (CTL4) (Panel F) on P. yoelii infection. The dots represent the number of oocysts present on individual midguts 6 days post infection. The median number of oocysts is indicated by the horizontal line. Distributions are compared using the KolmogorovSmirnov test; $\mathrm{n}=$ number of mosquitoes; $\mathrm{P}$ values lower than 0.05 are consider to be significantly different.

It has been proposed that $P$. falciparum parasites have evolved specific mechanisms to modulate activation of the An. gambiae immune system as they adapted to their natural mosquito vector $[23,24]$. The observation that $P$. falciparum strains from the New World, such as the Brazil- ian $P$. falciparum 7G8 strain, are melanized very effectively by the An. gambiae L35 strain but not those of African origin [9] adds support to the adaptation hypothesis. Recent experiments revealed that LRIM1 can also mediate immune responses against $P$. falciparum, because silencing 


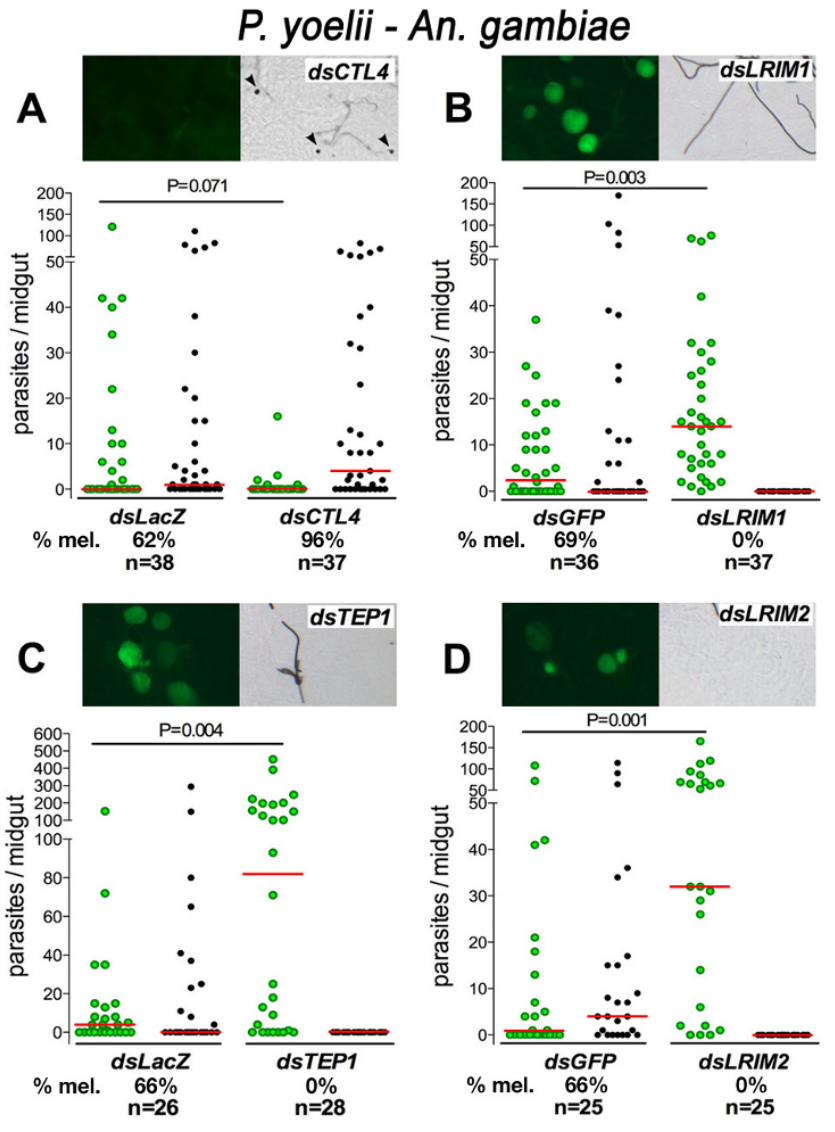

Figure 5

Effect of silencing An. gambiae (G3) CTL4, LRIMI, TEPI, or LRIM2 on P. yoelii infection. The images illustrate the level of infection and parasite melanization observed 6 days post infection (PI) when each gene was silenced. Live parasites are detected with green fluorescence (left panels) and those melanized are in DIC images (right panels). Effect of silencing C-type lectin 4 (CTL4) (Panel A), leucine rich-repeat immune protein I (LRIMI) (Panel B), thioester-containing protein I (TEPI) (Panel C), or leucine rich-repeat immune protein 2 (LRIM2) (Panel D) on P. yoelii infection. The dots represent the number of live (green) or melanized (black) parasites on individual midguts 6 days PI. The median number of oocysts is indicated by the horizontal line. Distributions are compared using the KolmogorovSmirnov test; $\mathrm{n}=$ number of mosquitoes; $\mathrm{P}$ values lower than 0.05 are consider to be significantly different.

this gene in An. gambiae L35 females infected with the Brazilian $P$. falciparum 7G8 strain completely reverts the melanization phenotype and results in live oocysts (A. Molina-Cruz, A and C. Barillas-Mury, unpublished). Selection for refractoriness to $P$. cynomolgy resulted in a strain of An. gambiae that is also refractory to multiple Plasmodium species. LRIM1 also mediates the antiparasitic responses of Anopheles quadriannulatus to P. berghei infection [25]. These findings indicate that some genes, such as
TEP1/LRIM1, are broad mediators of antiparasitic responses against several different Plasmodium parasites in different mosquito strains.

Under natural conditions, $P$. falciparum parasites must avoid or withstand the antiparasitic responses of An. gambiae to complete their life cycle and this is likely to exert selective pressure on parasite populations. For example, in Southern Mexico, three genetically distinct $P$. vivax populations have been identified, and experimental infections indicate that parasites are most compatible with sympatric mosquito species [26]. The authors propose that reciprocal selection between malaria parasites and mosquito vectors has led to local adaptation of parasites to their vectors [26]. Thus, it is likely that in well-adapted systems there is some level of immune evasion and/or suppression, and this could explain why silencing some genes involved in immunity (LRIM1, CTL4) or oxidative stress (OXR1, GSTT1 and GSTT2) in An. gambiae (G3) females, has little effect on $P$. falciparum (3D7 strain) infection.

There is also increasing evidence from many different studies that the interaction between Plasmodium parasites and the mosquito immune system it is a strong determinant of vectorial capacity. Nevertheless, the extent to which the mosquito immune system is effectively reducing Plasmodium infection is very variable, even between particular parasite and mosquito strains. These differences in compatibility need to be evaluated and carefully considered when selecting an experimental animal model to study malaria transmission.

\section{Methods \\ Mosquito rearing}

An. gambiae (G3 strain) and An. stephensi (Nijmegen Sda500) mosquitoes were raised at $28^{\circ} \mathrm{C}, 75 \%$ humidity under a 12-hour light/dark cycle and maintained on a $10 \%$ sucrose solution during adult stages.

\section{P. berghei and P. yoelii yoelii GFP I7XNL infections}

Either wild-type or GFP-P. berghei (ANKA 2.34 strain) [27] and the GFP-P. yoelii yoelii $17 \mathrm{X}$ nonlethal transgenic strain [28] were maintained by serial passage in 3-to 4-week-old female BALB/c mice or as frozen stocks. Mice parasitemias were monitored by light microscopy using air-dried blood smears that were methanol fixed and stained with $10 \%$ Giemsa. Female mosquitoes (4-5 days old) were fed on gametocytemic mice 2-3 days after blood inoculation from infected donor mice when parasitemias were between 5-10\%. Mosquitoes infected with $P$. berghei or $P$. yoelii were kept at $21^{\circ} \mathrm{C}$ or $24^{\circ} \mathrm{C}$, respectively, and midguts dissected 6-7 days post infection. Infection levels were determined by fluorescent (live oocyst) and light (melanized parasites) microscopy. The distribution of oocyst numbers in the different experimental groups was 
compared using the nonparametric Kolmogorov-Smirnov statistical test.

\section{Mosquito midgut genomic DNA extraction for quantitative real-time PCR (qPCR)}

Individual midguts (without blood) were placed into microcentrifuge tubes containing $10 \mu \mathrm{l}$ of HotSHOT alkaline lysis reagent ( $25 \mathrm{mM} \mathrm{NaOH}, 0.2 \mathrm{mM}$ EDTA, $\mathrm{pH} 12.0$ ) [29]. The tubes were boiled for $5 \mathrm{~min}$ and immediately placed on ice; $10 \mu \mathrm{l}$ of HotSHOT neutralizing reagent (40 $\mathrm{mM}$ Tris- $\mathrm{HCl}, \mathrm{pH}$ 5.0) was added to each tube. The samples were centrifuged and stored at $-20^{\circ} \mathrm{C}$.

\section{Determination of $P$. berghei infection by $q P C R$}

For the GSTT1 silencing experiment, mice were infected wild-type P. berghei (non-GFP parasites, Anka 2.34 parasites), and the level of infection in mosquitoes was determined by qPCR 6 days post infection. Genomic DNA was obtained from infected midguts, and the abundance of $P$. berghei 28S RNA relative to An. gambiae S7 ribosomal protein was determined. The DyNAmo SYBR Green qPCR Master mix (Finnzymes, Espoo, Finland) was used to amplify the genomic DNA, and samples were run on a MJ Research Detection system according to the manufacturer's instructions (Bio-Rad, Hercules, CA). P. berghei $28 \mathrm{~S}$ RNA primer sequence (5/ to 3/), Fw-GTGGCCTATCGATCCTTTA and Rev: 5/GCGTCCCAATGA TAGGAAGA). Two $\mu$ l of midgut genomic DNA was used to detect the number $P$. berghei $28 \mathrm{~S}$ gene copies and $1 \mu \mathrm{l}$ to determine the copies of An. gambiae ribosomal protein S7 gene in a $20-\mu$ l PCR reaction. All $P$. berghei 28 S values shown were then normalized relative to the number of copies of S7 in the sample. The distribution of parasite/midgut genome in control ( $d s L a c Z$ injected) and $d s G S T T 2$ silenced were compared using the Kolmogorov-Smirnov test.

\section{Experimental infection of An. gambiae mosquitoes with P. falciparum}

An. gambiae (G3) female mosquitoes were infected with $P$. falciparum by feeding them gametocyte cultures using an artificial membrane feeding system. The $P$. falciparum (3D7 strain) was maintained in O+ human erythrocytes using RPMI 1640 medium supplemented with $25 \mathrm{mM}$ HEPES, $50 \mathrm{mg} / \mathrm{L}$ hypoxanthine, $25 \mathrm{mM} \mathrm{NaHCO}$, and $10 \%(\mathrm{v} / \mathrm{v})$ heat-inactivated type $\mathrm{O}+$ human serum $[30,31]$. Gametocytogenesis was induced following the procedure of Ifediba and Vanderberg [32]. Mature gametocyte cultures (stages IV and V) that were 14-16 days old were used to feed mosquitoes in $37^{\circ} \mathrm{C}$ warmed membrane feeders for 30 minutes. To determine the level of infection, the midguts were dissected and stained with $0.05 \%(\mathrm{w} / \mathrm{v})$ mercurochrome in water and oocysts counted by light microscopy 7-9 days post blood feeding. Distribution of oocyst numbers per midgut was analyzed using the Kolmogorov-Smirnov test.

\section{dsRNA synthesis}

cDNA fragments of 500-600 bp were amplified for each gene using the primers shown in Additional File 1 and cDNA from 4-day-old An. gambiae females as template. The cDNA fragments were cloned into the $\mathrm{pCR}$ II-TOPO ${ }^{\circledR}$ vector (Invitrogen, Carlsbad, CA) and T7 sites introduced at both ends using the following vector primers (5' to 3') to amplify the cDNA insert; M13-Fw: GTAAAACGACGGCCAGT and T7-M13Rev: CTCGAGTAATACGACTCACTA TAGGGCAGGAAACAGCTATGAC. dsRNA was synthesized and purified using the MEGAscript kit (Ambion, Austin, TX). The eluted dsRNA was further cleaned and concentrated to $3 \mu \mathrm{g} / \mu \mathrm{l}$ using a Microcon YM100 filter (Millipore, Bedford, MA).

\section{Silencing An. gambiae genes}

dsRNA (207 ng in $69 \mathrm{nl}$ ) for each of the genes tested was injected into the thorax of cold-anesthetized 1- to 2-dayold female mosquitoes using a nano-injector (Nanoject; Drummond Scientific, Broomall, PA). In each experiment, a control group was injected with $d s L a c Z$ or $d s G F P$ to serve as reference for intensity of infection. Gene silencing was confirmed 4 days after dsRNA injection by RTqPCR using the ribosomal S7 gene for normalization. Poly(A) mRNA was isolated from groups of 10 adult females using Oligotex-dT beads (Qiagen, Valencia, CA) following the manufacturer's instructions. First-strand cDNA was synthesized using random hexamers and Superscript II reverse transcriptase (Invitrogen). The primers used for each gene are shown in Additional File 2. Gene expression was assessed by SYBR green qPCR (DyNAmo HS; New England Biolabs, Beverly, MA) in a Chromo4 system (Bio-Rad). PCR involved an initial denaturation at $95^{\circ} \mathrm{C}$ for 15 minutes, 44 cycles of 10 seconds at $94^{\circ} \mathrm{C}, 20$ seconds at $58^{\circ} \mathrm{C}$, and 30 seconds at $72^{\circ} \mathrm{C}$. Fluorescence readings were taken at $72^{\circ} \mathrm{C}$ after each cycle. A final extension at $72^{\circ} \mathrm{C}$ for 5 minutes was completed before deriving a melting curve $\left(70^{\circ} \mathrm{C}-95^{\circ} \mathrm{C}\right)$ to confirm the identity of the PCR product. qPCR measurements were made in duplicate.

\section{Silencing An. stephensi genes}

Because all the genes tested are highly conserved across species, we tested whether it was possible to silence An. stephensi genes by injecting them with dsRNA from orthologous genes of An. gambiae. An. stephensi female mosquitoes (1-2 days old) were injected with dsRNA from $A n$. gambiae cDNAs following the same procedure described above. Silencing efficiency was determined using qPCR 4 days after mosquitoes were injected with dsRNA. For the initial evaluation, the same primers and conditions as for An. gambiae were used, except for a lower annealing temperature $\left(52^{\circ} \mathrm{C}\right.$ instead of $\left.58^{\circ} \mathrm{C}\right)$. For $O X R 1$, a strong peak was obtained using the same primers as for An. gambiae, but for all other genes, several primer combinations from 
well conserved regions had to be designed to obtain efficient amplification that generated a single band of the expected molecular weight. For GSTT1, in was necessary to clone a fragment of An stephensi cDNA using the following degenerate primers (5/ to 3/), Fwd: CTGGCGGAAAGT GTKGCCAT and Rev: GGCCGCAGCCASACGTACTGGAA. A 180-bp fragment was amplified, sequenced, and used to generate a primer combination that would efficiently amplify AsGSTT1. Sequences of all primer sets used for qRT-PCR analysis with An. stephensi templates are shown in Additional File 3. Silencing efficiency in An. gambiae and An. stephensi, shown in Additional File 4, ranged from $55-98 \%$ and from $56-84 \%$, respectively.

\section{Abbreviations}

ADP: adenosine diphosphate; APL1: Anopheles Plasmodium-responsive leucine-rich repeat 1; $\operatorname{ArgK}$ : arginine kinase; ATP: adenosine triphosphate; cDNA: complimentary DNA; CTL4: C-type lectin 4; DIC: differential interference contrast; $d s A r g K, \operatorname{ArgK}$ dsRNA-injected mosquitoes; dsCTL4: C-type lectin 4 dsRNA-injected mosquitoes; $d s G F P$, GFP dsRNA-injected mosquitoes; dsGSTT1: glutathione-S-transferase theta 1 dsRNA-injected mosquitoes; dsGSTT2: glutathione-S-transferase theta 2 dsRNAinjected mosquitoes; $d s H s c-3$ : heat-shock cognate3dsRNA-injected mosquitoes; dsLacZ: $\beta$-galactosidasedsRNA-injected mosquitoes; $d s L R I M 1$ : leucine-rich repeat immune protein 1 dsRNA-injected mosquitoes; dsLRIM2: leucine-rich repeat immune protein 2 dsRNA-injected mosquitoes; dsOXR1: oxidation resistance 1 dsRNA-injected mosquitoes; dsRNA: double-stranded RNA; dsSol.trsp: solute transporter dsRNA-injected mosquitoes; dsTEP1: thioester-containing protein 1 dsRNA-injected mosquitoes; dsTetrasp: tetraspanin dsRNA-injected mosquitoes; GFP-P. yoelii yoelii 17XNL: Plasmodium yoelii yoelii 17X nonlethal transgenic strain constitutively expressing green fluorescent protein; GSTT: gene family, glutathione-Stransferase of the theta class gene family; GSTT1: glutathione-S-transferase theta 1 ; GSTT2: glutathione-S-transferase theta 2; Hsc-3: heat-shock cognate-3; KS: Kolmogorov-Smirnov; LRIM1: leucine-rich repeat immune protein 1; LRIM2: leucine-rich repeat immune protein 2; mRNA: messenger RNA; OXR1: oxidation resistance 1; PCR: polymerase chain reaction; qPCR: quantitative real-time PCR; qRT-PCR: quantitative real-time reverse-transcriptase PCR; ROS: reactive oxygen species; RPMI: Royal Park Memorial Institute; S7, protein from the small ribosomal subunit S7; Sol.trsp: solute transporter; TEP1: thioester-containing protein 1; Tetrasp: tetraspanin; TM: transmembrane domain.

\section{Authors' contributions}

GJ-G carried out most of the experimental work, data analysis, and drafted the manuscript. JR performed most of the experiments involving silencing of GSTT1 and helped with midgut dissections and oocyst counting. GN and GJ-G performed the P. yoelii infections in An. gambiae and An. stephensi. MP and GJ-G silenced TEP1, LRIM1, and LRIM2 in P. yoelii-infected An. gambiae. A M-C prepared the $P$. falciparum gametocyte cultures. C B-M contributed with experimental design, data analysis, image processing, assembly of final figures, and writing the manuscript.

\section{Additional material}

\section{Additional file 1}

Validation of gene silencing in An. gambiae and An. stephensi. The data indicate the silencing efficiency of several genes after dsRNA injection in An. gambiae and An. stephensi, relative to a control group injected with dsLacZ.

Click here for file

[http://www.biomedcentral.com/content/supplementary/14712180-9-154-S1.pdf]

\section{Additional file 2}

Primers used to generate dsRNA using An. gambiae cDNA as template. The data indicate the sequence of the primers used to generate dsRNA using An. gambiae cDNA as template.

Click here for file

[http://www.biomedcentral.com/content/supplementary/14712180-9-154-S2.pdf]

\section{Additional file 3}

Primers used to determine gene expression by $q R T-P C R$ and validate gene silencing in An. gambiae. The data indicate the sequence of the primers used for gene expression analysis by qRT-PCR to validate gene silencing in An. gambiae.

Click here for file

[http://www.biomedcentral.com/content/supplementary/14712180-9-154-S3.pdf]

\section{Additional file 4}

Primers used to determine gene expression by qRT-PCR and validate gene silencing in An. stephensi. The data indicate the sequence of the primers used for gene expression analysis by qRT-PCR to validate gene silencing in An. stephensi.

Click here for file

[http://www.biomedcentral.com/content/supplementary/14712180-9-154-S4.pdf]

\section{Acknowledgements}

We thank André Laughinghouse, Kevin Lee, Tovi Lehman, and Robert Gwadz for insectary support and NIAID Intramural editor Brenda Rae Marshall. This research was supported by the Intramural Research Program of the Division of Intramural Research National Institute of Allergy and Infectious Diseases, National Institutes of Health.

\section{References}

I. Blandin S, Shiao SH, Moita LF, Janse CJ, Waters AP, Kafatos FC, Levashina EA: Complement-like protein TEPI is a determinant of vectorial capacity in the malaria vector Anopheles gambiae. Cell 2004, I I 6(5):66 I-670. 
2. Osta MA, Christophides GK, Kafatos FC: Effects of mosquito genes on Plasmodium development. Science 2004, 303(5666):2030-2032.

3. Riehle MM, Markianos K, Niare O, Xu J, Li J, Toure AM, Podiougou B, Oduol F, Diawara S, Diallo $M$, et al.: Natural malaria infection in Anopheles gambiae is regulated by a single genomic control region. Science 2006, 3 I 2(5773):577-579.

4. Dong Y, Aguilar R, Xi Z, Warr E, Mongin E, Dimopoulos G: Anopheles gambiae immune responses to human and rodent $P$ lasmodium parasite species. PLoS Pathog 2006, 2(6):e52.

5. Tahar R, Boudin C, Thiery I, Bourgouin C: Immune response of Anopheles gambiae to the early sporogonic stages of the human malaria parasite Plasmodium falciparum. EMBO J 2002 2 I (24):6673-6680.

6. Cohuet A, Osta MA, Morlais I, Awono-Ambene PH, Michel K, Simard F, Christophides GK, Fontenille D, Kafatos FC: Anopheles and Plasmodium: from laboratory models to natural systems in the field. EMBO Rep 2006, 7(I2): | 285-1289.

7. Service MW: Community participation in vector-borne disease control. Ann Trop Med Parasitol 1993, 87(3):223-234.

8. Feldmann AM, Ponnudurai T: Selection of Anopheles stephensi for refractoriness and susceptibility to Plasmodium falciparum. Med Vet Entomol 1989, 3(I):41-52.

9. Collins FH, Sakai RK, Vernick KD, Paskewitz S, Seeley DC, Miller LH, Collins WE, Campbell CC, Gwadz RW: Genetic selection of a Plasmodium-refractory strain of the malaria vector Anopheles gambiae. Science 1986, 234(4776):607-610.

10. Lambrechts L, Halbert J, Durand P, Gouagna LC, Koella JC: Host genotype by parasite genotype interactions underlying the resistance of anopheline mosquitoes to Plasmodium falciparum. Malar J 2005, 4:3.

II. Schneider D, Shahabuddin M: Malaria parasite development in a Drosophila model. Science 2000, 288(5475):2376-2379.

12. Brandt SM, Jaramillo-Gutierrez G, Kumar S, Barillas-Mury C, Schneider DS: Use of a Drosophila Model to Identify Genes Regulating Plasmodium Growth in the Mosquito. Genetics 2008, |80(3): |67|-|678.

13. Chamberlin M: Mitochondrial arginine kinase in the midgut of the tobacco hornworm (Manduca sexta). J Exp Biol 1997, 200(Pt 2 I ):2789-2796.

14. Hemler ME: Tetraspanin functions and associated microdomains. Nat Rev Mol Cell Biol 2005, 6( I 0):80 I-8I I.

15. Silvie O, Rubinstein E, Franetich JF, Prenant M, Belnoue E, Renia L, Hannoun L, Eling W, Levy S, Boucheix C, et al.: Hepatocyte CD8 I is required for Plasmodium falciparum and Plasmodium yoelii sporozoite infectivity. Nat Med 2003, 9(I):93-96.

16. Elliott NA, Volkert MR: Stress induction and mitochondrial localization of Oxrl proteins in yeast and humans. Mol Cell Biol 2004, 24(8):3 I80-3 I87.

17. Molina-Cruz A, Dejong RJ, Charles B, Gupta L, Kumar S, JaramilloGutierrez G, Barillas-Mury C: Reactive oxygen species modulate Anopheles gambiae immunity against bacteria and Plasmodium. J Biol Chem 2008, 283(6):32 I7-3223.

18. Ding Y, Ortelli F, Rossiter LC, Hemingway J, Ranson H: The Anopheles gambiae glutathione transferase supergene family: annotation, phylogeny and expression profiles. BMC Genomics 2003, 4(I):35.

19. Lear JT, Heagerty AH, Smith A, Bowers B, Payne CR, Smith CA, Jones PW, Gilford J, Yengi L, Alldersea J, et al.: Multiple cutaneous basal cell carcinomas: glutathione S-transferase (GSTMI, GSTTI) and cytochrome P450 (CYP2D6, CYPIAI) polymorphisms influence tumour numbers and accrual. Carcinogenesis 1996, I7(9): $1891-1896$.

20. Hayek T, Stephens JW, Hubbart CS, Acharya J, Caslake MJ, Hawe E, Miller GJ, Hurel SJ, Humphries SE: A common variant in the glutathione $S$ transferase gene is associated with elevated markers of inflammation and lipid peroxidation in subjects with diabetes mellitus. Atherosclerosis 2006, I 84(2):404-4I2.

21. Vaughan JA, Hensley L, Beier JC: Sporogonic development of Plasmodium yoelii in five anopheline species. J Parasitol 1994 , 80(5):674-68I.

22. Garver LS, Dong Y, Dimopoulos G: Caspar controls resistance to Plasmodium falciparum in diverse anopheline species. PLOS Pathog 2009, 5(3):el 000335.

23. Michel K, Suwanchaichinda C, Morlais I, Lambrechts L, Cohuet A, Awono-Ambene PH, Simard F, Fontenille D, Kanost MR, Kafatos FC:
Increased melanizing activity in Anopheles gambiae does not affect development of Plasmodium falciparum. Proc Natl Acad Sci USA 2006, I03(45): I6858-16863.

24. Lambrechts L, Morlais I, Awono-Ambene PH, Cohuet A, Simard F, Jacques JC, Bourgouin C, Koella JC: Effect of infection by Plasmodium falciparum on the melanization immune response of Anopheles gambiae. Am J Trop Med Hyg 2007, 76(3):475-480.

25. Habtewold T, Povelones M, Blagborough AM, Christophides GK: Transmission blocking immunity in the malaria non-vector mosquito Anopheles quadriannulatus species A. PLoS Pathog 2008, 4(5): el 000070.

26. Joy DA, Gonzalez-Ceron L, Carlton JM, Gueye A, Fay M, McCutchan TF, Su XZ: Local adaptation and vector-mediated population structure in Plasmodium vivax malaria. Mol Biol Evol 2008 , 25(6): | 245- | 252

27. Franke-Fayard B, Trueman H, Ramesar J, Mendoza J, Keur M van der, Linden R van der, Sinden RE, Waters AP, Janse C): A Plasmodium berghei reference line that constitutively expresses GFP at a high level throughout the complete life cycle. Mol Biochem Parasitol 2004, I37(I):23-33

28. Ono T, Tadakuma T, Rodriguez A: Plasmodium yoelii yoelii I7XNL constitutively expressing GFP throughout the life cycle. Exp Parasitol 2007, I | 5(3):3 | 0-3 | 3

29. Truett GE, Heeger P, Mynatt RL, Truett AA, Walker JA, Warman ML: Preparation of PCR-quality mouse genomic DNA with hot sodium hydroxide and tris (HotSHOT). Biotechniques 2000, 29(I):52. 54.

30. Trager $\mathrm{W}$, Jensen JB: Human malaria parasites in continuous culture. Science 1976, 193(4254):673-675.

31. Zolg JW, MacLeod AJ, Dickson IH, Scaife JG: Plasmodium falciparum: modifications of the in vitro culture conditions improving parasitic yields. J Parasitol I 982, 68(6): | 072- 1080.

32. Ifediba T, Vanderberg JP: Complete in vitro maturation of Plasmodium falciparum gametocytes. Nature 1981, 294(5839):364-366.
Publish with Biomed Central and every scientist can read your work free of charge

"BioMed Central will be the most significant development for disseminating the results of biomedical research in our lifetime. "

Sir Paul Nurse, Cancer Research UK

Your research papers will be:

- available free of charge to the entire biomedical community

- peer reviewed and published immediately upon acceptance

- cited in PubMed and archived on PubMed Central

- yours - you keep the copyright
BioMedcentral 